\title{
Antifungal treatment for invasive Candida infections: a mixed treatment comparison meta-analysis
}

\author{
Edward J Mills*1,2, Dan Perri ${ }^{3}$, Curtis Cooper ${ }^{4}$, Jean B Nachega ${ }^{5,6}$, Ping Wu ${ }^{2}$, \\ Imad Tleyjeh ${ }^{7,8}$ and Peter Phillips 9
}

\begin{abstract}
Address: ${ }^{1}$ Faculty of Health Sciences, Simon Fraser University, Burnaby, Canada, ${ }^{2}$ Department of Clinical Epidemiology \& Biostatistics, McMaster University, Hamilton, Canada, ${ }^{3}$ Department of Medicine, McMaster University, Hamilton, Canada, ${ }^{4}$ Division of Infectious Diseases, Ottawa Hospital, University of Ottawa, Ottawa, Canada, ${ }^{5}$ Departments of Epidemiology and International Health, Johns Hopkins Bloomberg School of Public Health, Baltimore, Maryland, USA, ${ }^{\circ}$ Department of Medicine and Centre for Infectious Diseases, Faculty of Health Sciences, Stellenbosch University, Cape Town, South Africa, ${ }^{7}$ Division of Infectious Diseases, Department of Medicine, Research Center, King Fahd Medical City, Riyadh, Saudi Arabia, ${ }^{8}$ Division of Infectious Diseases, Department of Medicine, Mayo Clinic, Rochester, MN, USA and ${ }^{9}$ Division of Infectious Diseases, University of British Columbia, Vancouver, Canada
\end{abstract}

Email: Edward J Mills* - emills@cfenet.ubc.ca; Dan Perri - dan.perri@utoronto.ca; Curtis Cooper - ccooper@ottawahospital.on.ca; Jean B Nachega - jnachega@jhsph.edu; Ping Wu - pwu@ccnm.edu; Imad Tleyjeh - Tleyjeh.Imad@mayo.edu;

Peter Phillips - pphillips@cfenet.ubc.ca

* Corresponding author

Published: 26 June 2009

Annals of Clinical Microbiology and Antimicrobials 2009, 8:23 doi:10.1 I86/1476-07। I-8-23

This article is available from: http://www.ann-clinmicrob.com/content/8/I/23

(C) 2009 Mills et al; licensee BioMed Central Ltd.

This is an Open Access article distributed under the terms of the Creative Commons Attribution License (http://creativecommons.org/licenses/by/2.0), which permits unrestricted use, distribution, and reproduction in any medium, provided the original work is properly cited.
Received: 2I January 2009

Accepted: 26 June 2009

Objectives: Invasive fungal infections are a major cause of mortality among patients at risk. Treatment guidelines vary on optimal treatment strategies. We aimed to determine the effects of different antifungal therapies on global response rates, mortality and safety.

Methods: We searched independently and in duplicate 10 electronic databases from inception to May 2009. We selected any randomized trial assessing established antifungal therapies for confirmed cases of invasive candidiasis among predominantly adult populations. We performed a meta-analysis and then conducted a Bayesian mixed treatment comparison to differentiate treatment effectiveness. Sensitivity analyses included dosage forms of amphotericin B and fluconazole compared to other azoles.

Results: Our analysis included II studies enrolling a total of 965 patients. For our primary analysis of global response rates, we pooled 7 trials comparing azoles to amphotericin B, Relative Risk [RR] 0.87 (95\% Confidence Interval $[\mathrm{Cl}], 0.78-0.96, P=0.007, \mathrm{I}^{2}=43 \%, \mathrm{P}=0.09$. We also pooled 2 trials of echinocandins versus amphotericin $\mathrm{B}$ and found a pooled $\mathrm{RR}$ of $\mathrm{I} .10(95 \% \mathrm{Cl}, 0.99-\mathrm{I} .23, \mathrm{P}=0.08)$. One study compared anidulafungin to fluconazole and yielded a RR of $\mathrm{I} .26(95 \% \mathrm{Cl}, 1.06-\mathrm{I} .5 \mathrm{I})$ in favor of anidulafungin. We pooled 7 trials assessing azoles versus amphotericin $B$ for all-cause mortality, resulting in a pooled RR of $0.88\left(95 \% \mathrm{Cl}, 0.74-1.05, \mathrm{P}=0.17, \mathrm{I}^{2}=0 \%, \mathrm{P}=0.96\right)$. Echinocandins versus amphotericin $\mathrm{B}(2$ trials $)$ for allcause mortality resulted in a pooled $\mathrm{RR}$ of $\mathrm{I} .0 \mathrm{I}(95 \% \mathrm{Cl}, 0.84-\mathrm{I} .20, \mathrm{P}=0.93)$. Anidulafungin versus fluconazole resulted in a RR of $0.73(95 \% \mathrm{Cl}, 0.48-\mathrm{I} .10, \mathrm{P}=0.34)$. Our mixed treatment comparison analysis found similar within-class effects across all interventions. Adverse event profiles differed, with amphotericin B exhibiting larger adverse event effects.

Conclusion: Treatment options appear to offer preferential effects on response rates and mortality. When mycologic data are available, therapy should be tailored. 


\section{Introduction}

Invasive fungal infections contribute importantly to morbidity and mortality in immunocompromised patients including those with hematologic cancers, recent transplants, autoimmune disorders, and critical illness. The most common fungal pathogen is Candida.

For a number of reasons, early and accurate diagnosis of invasive fungal infections is often difficult and patients from these high risk groups may have evidence of disseminated fungal infection at autopsy that was not identified prior to death[1]. Clinical manifestations of invasive fungal infections often occur at a late stage of infection contributing to diagnostic delays and higher case-fatality rates. Immunocompromised patients may not generate detectable antibody responses to specific fungal pathogens[2]. Furthermore, non-invasive antigen detection methods such as the Fungitec $\mathrm{G}$ assay for beta-1-3 glucan are not widely available. Finally, culture techniques are not highly sensitive, and invasive diagnostic techniques may be contraindicated or not applicable to the clinical presentation. Consequently, antifungal treatment for confirmed invasive fungal infections is challenging and evaluations of therapeutic interventions are limited[3]. Several choices of antifungal agents exist that differ greatly with respect to both toxicity and cost [4].

There is an ever-growing literature on the use of antifungal agents in patients with candidemia. Previous systematic reviews have not looked at the relative effectiveness of interventions of confirmed infections [4-6]. Using a systematic review of the literature and meta-analytic techniques, we aimed to quantify the effects of antifungal therapy on confirmed systemic fungal infection response rates, associated mortality and safety when reserved for confirmed cases only. Furthermore, we determined differences in treatment effects across interventions using a mixed treatment comparison meta-analysis.

\section{Methods}

\section{Eligibility criteria}

We included any randomized trial of antifungal therapies for confirmed cases of invasive candidiasis among predominantly adult ( $\geq 18$ years of age) populations. We included randomized trials of any duration. Given that head to head evaluations have existed for decades, studies had to compare antifungal therapy to another antifungal therapy (head-to-head evaluations). Studies had to report on any of the following clinically-important outcomes: clinical response, all-cause mortality; fungal-attributable death, and adverse events. We excluded studies only reporting on dose-comparison or dosage form evaluations. As we were interested in disseminated disease, trials focused on single site fungal infections (mucocutaneous, esophageal, dermatologic, meningeal, bladder, or focal chest) were excluded, as were aspergillosis trials, cryptococcosis and endemic mycoses trials. We excluded drugs no longer recommended by Infectious Disease Society of America Guidelines (IDSA) including ketoconazole[7].

\section{Search strategy}

In consultation with a medical librarian, we (DP, EM) established a search strategy (available from corresponding author on request). We searched independently, in duplicate, the following 10 databases (from inception to May 2009): MEDLINE, EMBASE, Cochrane CENTRAL, AMED, CINAHL, TOXNET, Development and Reproductive Toxicology, Hazardous Substances Databank, Psychinfo and Web of Science, databases that included the full text of journals (OVID, ScienceDirect, and Ingenta, including articles in full text from approximately 1700 journals since 1993). Key search words included words addressing the infections: Fungus, fungal, fungemia, mycosis, candidiasis, candidemia, candida; and words addressing the interventions: antifungal, amphotericin, azoles, triazoles, fluconazole, itraconazole, miconazole, voriconazole, posaconazole, ravuconazole, flucytosine, echinocandins, caspofungin, micafungin, anidulafungin, confirmed; and finally a word indicating a randomized trial: random* (wildcard). In addition, we searched the bibliographies of published systematic reviews and collected papers. We contacted the authors of trials for study clarifications, where required. Searches were not limited by language, sex or age.

\section{Study selection}

Two investigators (DP, EM) working independently, in duplicate, scanned all abstracts and obtained the full-text reports of records, that indicated or suggested that the study was a randomized trial evaluating antifungal therapy on the outcomes of interest. After obtaining full reports of the candidate trials, in full peer-reviewed publication, the same reviewers independently assessed eligibility from full text papers.

\section{Data collection}

The same 2 reviewers conducted data extraction independently using a standardized pre-piloted form. Reviewers collected information about the antifungal therapy and type of interventions tested, the population studied (age, setting, underlying conditions), the treatment effect on specified outcomes, adverse events, and specific adverse events addressing renal toxicity and liver impairment. Study evaluation included general methodological quality features, including allocation concealment, sequence generation, a description of who was blinded, use of intention-to-treat analysis and proportion of study population lost-to-follow-up. We entered the data into an electronic database such that duplicate entries existed for each study; when the two entries did not match, we resolved differences through discussion and consensus. 


\section{Data analysis}

In order to assess inter-rater reliability on inclusion of articles, we calculated the Phi statistic, that provides a measure of inter-observer agreement independent of chance[8]. Our primary outcome of interest is response rates. Secondary outcomes include all-cause mortality, fungal-attributable mortality, and adverse events.

We performed two specific analyses. First, we performed a frequentist fixed-effects meta-analysis of study outcomes across classes of drugs, applying a Relative Risk [RR] and appropriate 95\% Confidence Intervals [CIs] of outcomes according to the number of events reported in the original studies. In the event of zero outcome events in one arm of a trial, we used the Haldane method and added 0.5 to each arm[9]. Given the varied size of studies, we pooled studies using a fixed effects approach that recognizes the precision of studies and provides greater to weight to larger studies[10]. We calculated the $\mathrm{I}^{2}$ statistic for each analysis as a measure of the proportion of the overall variation that is attributable to between-study heterogeneity[11]. Given the varied interventions, and the consideration that most trials were not no-treatment or placebo controlled, we pooled studies assessing withinclass interventions as head-to-head trials. Our first analysis examined drugs within-class and then examined individual drugs using the mixed treatment comparisons. We initially pooled all azole interventions versus all amphotericin B trials for response and conducted a multivariable meta-regression using the unrestricted maximum likelihood method assessing the impact of individual azoles on the overall estimate of effect and the individual delivery methods of amphotericin B on overall estimate[12]. Our regression covariates were chosen a priori and included: amphotericin delivery and allocation concealment. Analyses were conducted using StatsDirect and STATA.

For our second analysis, we examined the relative effectiveness of each individual drug using the Lu-Ades fixed effects method for combining direct and indirect evidence in mixed treatment comparisons, a Bayesian approach[13]. We estimated the posterior densities for all unknown parameters using MCMC (Markov chain Monte Carlo) for each model in WinBUGS version 1.4 (Medical Research Council Biostatistics Unit, Cambridge). Each chain used 100,000 iterations with a burn-in of 500, thin of 5 , and updates varying between 80 and 110 . We used the same seed number (SEED = 314159) for all chains. The choice of burn-in was chosen according to GelmanRubin approach[14]. We assessed convergence based on trace plots and time series plots (available upon request). The accuracy of the posterior estimates was done by calculating the Monte Carlo error for each parameter. As a rule of thumb, the Monte Carlo error for each parameter of interest is less than about $5 \%$ of the sample standard deviation[15]. All results are reported as posterior means with corresponding 95\% credibility intervals (CrIs). Credibility intervals are the Bayesian equivalent of classical confidence intervals.

\section{Results}

Our literature search identified 1284 potentially relevant abstracts of full text articles. Of these, 42 full text RCTs were obtained. We excluded 31, leaving 11 that met our

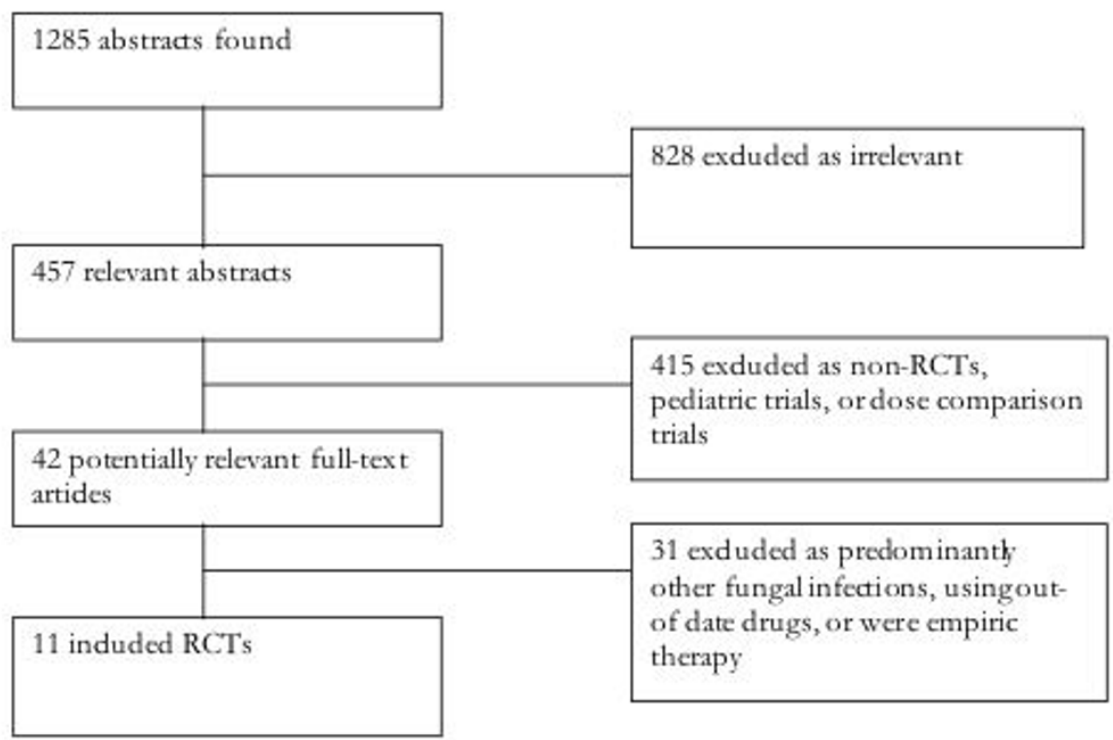

Figure I

Flow-diagram of search and included studies. 


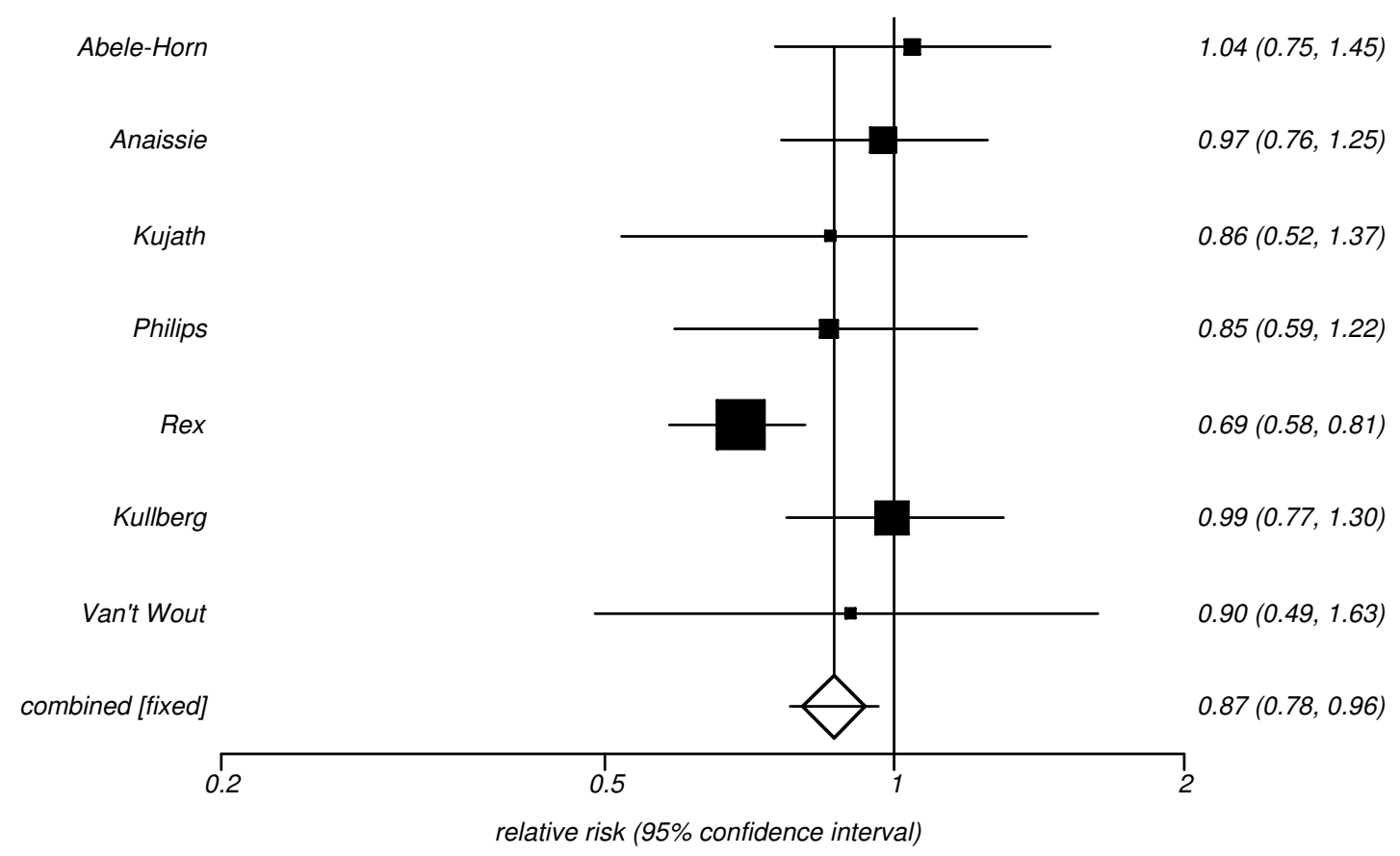

Figure 2

Global response rate fixed-effects meta-analysis: Triazoles versus amphotericin $B$.

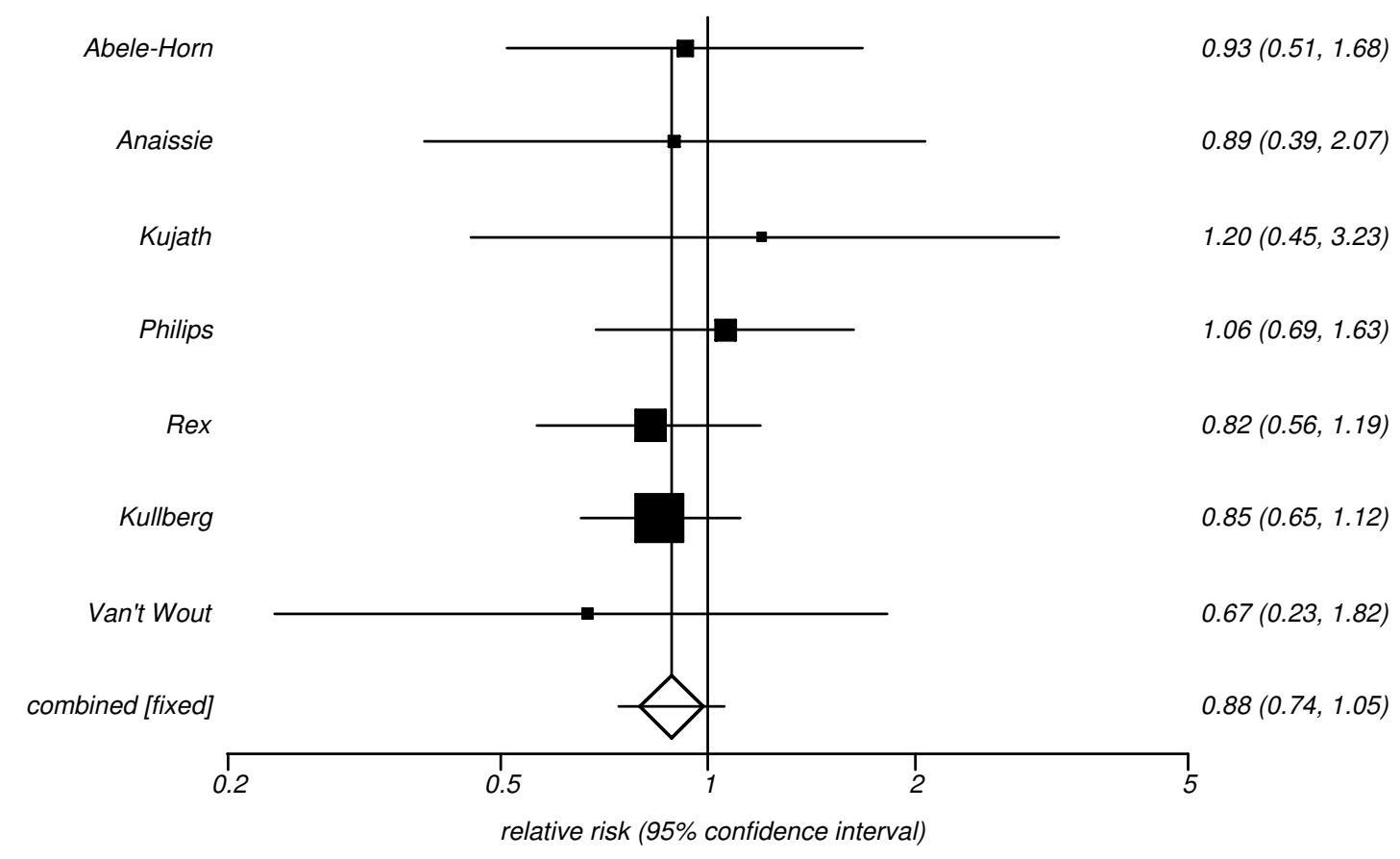

Figure 3

All-cause mortality fixed-effects meta-analysis: Triazoles versus amphotericin B. 
Table I: Odds ratios and $95 \%$ Crls for mixed treatment comparisons of confirmed infection studies, Response rates

\begin{tabular}{|c|c|c|}
\hline Treatment Comparison & Odds Ratio & 95\% Credible Interval \\
\hline Caspofungin vs. Fluconazole & 2.03 & $(0.98,3.76)$ \\
\hline Amphotericin B Deoxycholate vs. Fluconazole & 1.13 & $(0.78,1.58)$ \\
\hline Amphotericin B Liposomal vs. Fluconazole & 1.85 & $(0.65,4.19)$ \\
\hline Voriconazole vs. Fluconazole & 1.14 & $(0.62,1.94)$ \\
\hline Micafungin vs. Fluconazole & 2.13 & $(0.83,4.55)$ \\
\hline Anidulafungin vs. Fluconazole & 2.14 & $(1.19,3.58)$ \\
\hline Itraconazole vs. Fluconazole & 1.97 & $(0.32,6.69)$ \\
\hline Amphotericin B Deoxycholate vs. Caspofungin & 0.60 & $(0.32,1.02)$ \\
\hline Amphotericin B Liposomal vs. Caspofungin & 0.91 & $(0.45,1.63)$ \\
\hline Voriconazole vs. Caspofungin & 0.61 & $(0.27,1.18)$ \\
\hline Micafungin vs. Caspofungin & 1.04 & $(0.59,1.70)$ \\
\hline Anidulafungin vs. Caspofungin & 1.18 & $(0.45,2.56)$ \\
\hline Itraconazole vs. Caspofungin & 1.05 & $(0.16,3.72)$ \\
\hline Amphotericin B Liposomal vs. Amphotericin B Deoxycholate & 1.64 & $(0.63,3.52)$ \\
\hline Voriconazole vs. Amphotericin B Deoxycholate & 1.01 & $(0.63,1.54)$ \\
\hline Micafungin vs. Amphotericin B Deoxycholate & 1.88 & $(0.80,3.79)$ \\
\hline Anidulafungin vs. Amphotericin B Deoxycholate & 1.96 & $(0.96,3.58)$ \\
\hline Itraconazole vs. Amphotericin B Deoxycholate & 1.74 & $(0.30,5.77)$ \\
\hline Voriconazole vs. Amphotericin B Liposomal & 0.75 & $(0.25,1.74)$ \\
\hline Micafungin vs. Amphotericin B Liposomal & 1.18 & $(0.81,1.68)$ \\
\hline Anidulafungin vs. Amphotericin B Liposomal & 1.44 & $(0.42,3.66)$ \\
\hline Itraconazole vs. Amphotericin B Liposomal & 1.28 & $(0.16,4.86)$ \\
\hline Micafungin vs. Voriconazole & 1.95 & $(0.72,4.31)$ \\
\hline Anidulafungin vs. Voriconazole & 2.03 & $(0.85,4.13)$ \\
\hline Itraconazole vs. Voriconazole & 1.81 & $(0.28,6.22)$ \\
\hline Anidulafungin vs. Micafungin & 1.21 & $(0.38,2.94)$ \\
\hline Itraconazole vs. Micafungin & 1.08 & $(0.14,3.99)$ \\
\hline Itraconazole vs. Anidulafungin & 0.99 & $(0.14,3.56)$ \\
\hline
\end{tabular}

inclusion criteria[2,3,16-24], Phi $=0.91, \mathrm{n}=2,554$, See Figure 1 and Additional File 1 . The majority $(n=7)$ of studies assessed the head-to-head non-inferiority of azoleclass drugs compared to amphotericin B. The azoles include fluconazole $[2,3,17,18,23]$, itraconazole[16], and voriconazole[19]. The other studies assessed anidulafungin to fluconazole[22], micafungin to amphotericin $\mathrm{B}[20]$; caspofungin to amphotericin $\mathrm{B}[21]$; and micafungin to caspofungin[24]. Trials were predominantly conducted in populations dominated by patients with hematologic cancers experiencing infection with Candida species. The median participant age was 57 years (IQR 5659).

In keeping with the time periods that the studies were published (1991-2007), reporting of study methodological features was moderate. Seven of eleven trials reported on sequence generation; 6 of 11 on allocation concealment; 3 of 11 on who was blinded; 9 of 11 reported using intent-to-treat principles; and 6 of 11 provided details on participants lost-to-follow-up. An average of $10.8 \%$ of study participants were lost-to-follow-up.

\section{Meta-analysis}

Global response rates

For our primary outcome, we pooled 7 trials $(n=965$, See Figure 2) assessing azoles to amphotericin B. Our pooled estimate is 0.87 (95\% CI, 0.78-0.96, $\mathrm{P}=0.007, \mathrm{I}^{2}=43 \%$, $\mathrm{P}=0.09)$. When we compared only fluconazole trials (5 trials) to amphotericin $\mathrm{B}$, we found similar effects (RR $\left.0.82,95 \% \mathrm{CI}, 0.74-0.92, \mathrm{P}=0.0009, \mathrm{I}^{2}=52 \%, \mathrm{P}=0.07\right)$. The itraconazole versus amphotericin B trial (RR 0.90, 95\% CI, 0.49-1.63, $\mathrm{P}=0.61$ ) and voriconazole versus amphotericin B trial (RR 0.99, 95\% CI, 0.77-1.30, P = 0.94 ) provided similar estimates.

We also pooled 2 trials of echinocandins (micafungin[20] and caspofungin[21]) versus amphotericin B and found a 
Table 2: Absolute treatment efficacy and the probability that each treatment is best in the mixed treatment comparisons analysis using the response data from the confirmed infection studies.

\begin{tabular}{lcc}
\hline & \multicolumn{2}{c}{ Response Rates } \\
\hline Rluconazole & 63.00 & 0.000 \\
\hline Caspofungin & 76.10 & 0.139 \\
\hline Amphotericin B Deoxycholate & 65.40 & 0.000 \\
\hline Amphotericin B Liposomal & 72.98 & 0.070 \\
\hline Voriconazole & 65.03 & 0.004 \\
\hline Micafungin & 75.98 & 0.200 \\
\hline Anidulafungin & 77.49 & 0.345 \\
\hline Itraconazole & 69.33 & 0.241 \\
\hline
\end{tabular}

pooled RR of 1.10 (95\% CI, $0.99-1.23, \mathrm{P}=0.08)$. The anidulafungin to fluconazole trial yielded a RR of $1.26(95 \%$ CI, 1.06-1.51, $\mathrm{P}=0.001$ ) in favor of anidulafungin[22]; and micafungin to caspofungin (RR 1.00, 95\% CI, 0.94$1.08, \mathrm{P}=0.21)[24]$.

All-cause mortality

Our secondary outcomes included all-cause mortality. We pooled 7 trials $(n=965$, figure 3 ) assessing azoles versus amphotericin $\mathrm{B}$ for all-cause mortality, resulting in a pooled RR of 0.88 (95\% CI, 0.74-1.05, $\mathrm{P}=0.17, \mathrm{I}^{2}=0 \%$, $\mathrm{P}=0.96)$. This was also found when individual azoles were analyzed: fluconazole ( 5 trials) RR 0.92 (95\% CI, $0.73-1.17, \mathrm{P}=0.51, \mathrm{I}^{2}=0 \%, \mathrm{P}=0.90$; itraconazole ( 1 trials) RR $0.67,95 \% \mathrm{CI}, 0.74-1.05, \mathrm{P}=0.20$; voriconazole ( 1 trials) RR 0.85, 95\% CI, 0.65-1.12, $\mathrm{P}=0.67$ ).

When we assessed echinocandins versus amphotericin B preparations ( 2 trials) for all-cause mortality, we found a pooled RR of 1.01 (95\% CI, 0.84-1.20, $\mathrm{P}=0.93$ ). Micafungin versus caspofungin resulted in a RR of 0.85 (95\% CI, 0.96-1.11) in the direction of favour of caspofungin. Anidulafungin versus fluconazole resulted in a RR of $0.73(95 \% \mathrm{CI}, 0.48-1.10, \mathrm{P}=0.34)$ in the direction of anidulafungin.

\section{Fungal-attributable mortality}

We also assessed deaths attributable to the fungal infections. When we pooled 5 azole trials versus amphotericin $\mathrm{B}$, we found a pooled RR of 0.84 (95\% CI, $0.49-1.42, \mathrm{P}=$ $\left.0.51, \mathrm{I}^{2}=0 \%, \mathrm{P}=0.74\right)$. When we pooled the 3 echinocandin trials versus amphotericin $\mathrm{B}$, we found a pooled $\mathrm{RR}$ of 1.16 (95\% CI, $0.75-1.79, \mathrm{P}=0.50)$. Anidulafungin versus fluconazole yielded a RR of 0.84 (95\% CI, 0.48-1.47, $\mathrm{P}=0.88)$.

\section{Adverse events}

To assess serious adverse events, we pooled 2 trials of azoles versus amphotericin $\mathrm{B}$ assessing serious adverse events and found a pooled RR of 0.67 (95\% CI, 0.55$0.81, \mathrm{P}=<0.0001$ ) in favour of azoles. We also pooled 2 trials of echinocandins versus amphotericin $B$ and found

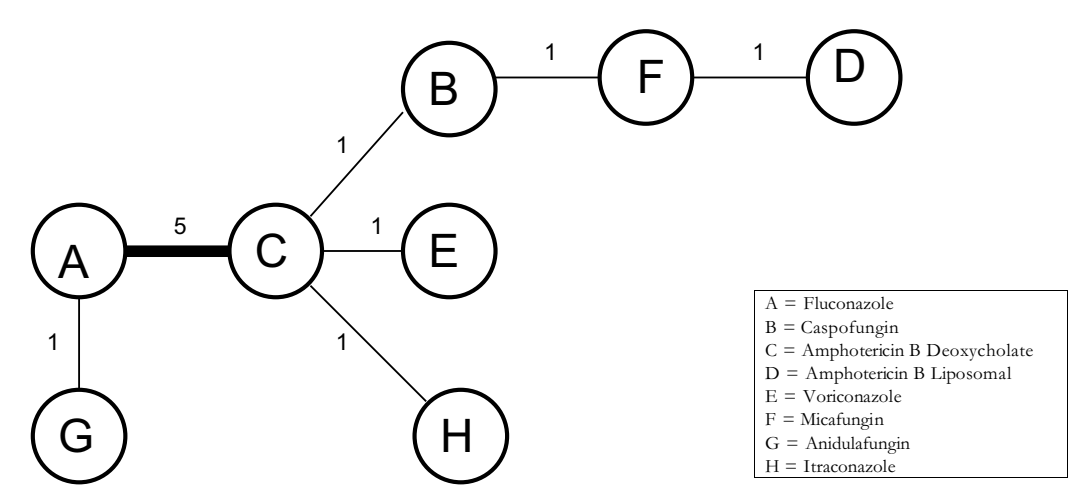

Figure 4

Network of evidence formed by the eight antifungal treatments compared on the basis of mortality data from I I studies. Each treatment is a node in the network. The links between nodes are trials or pairs of trial arms. The numbers along the link lines indicate the number of trials or pairs of trial arms for that link in the network. 


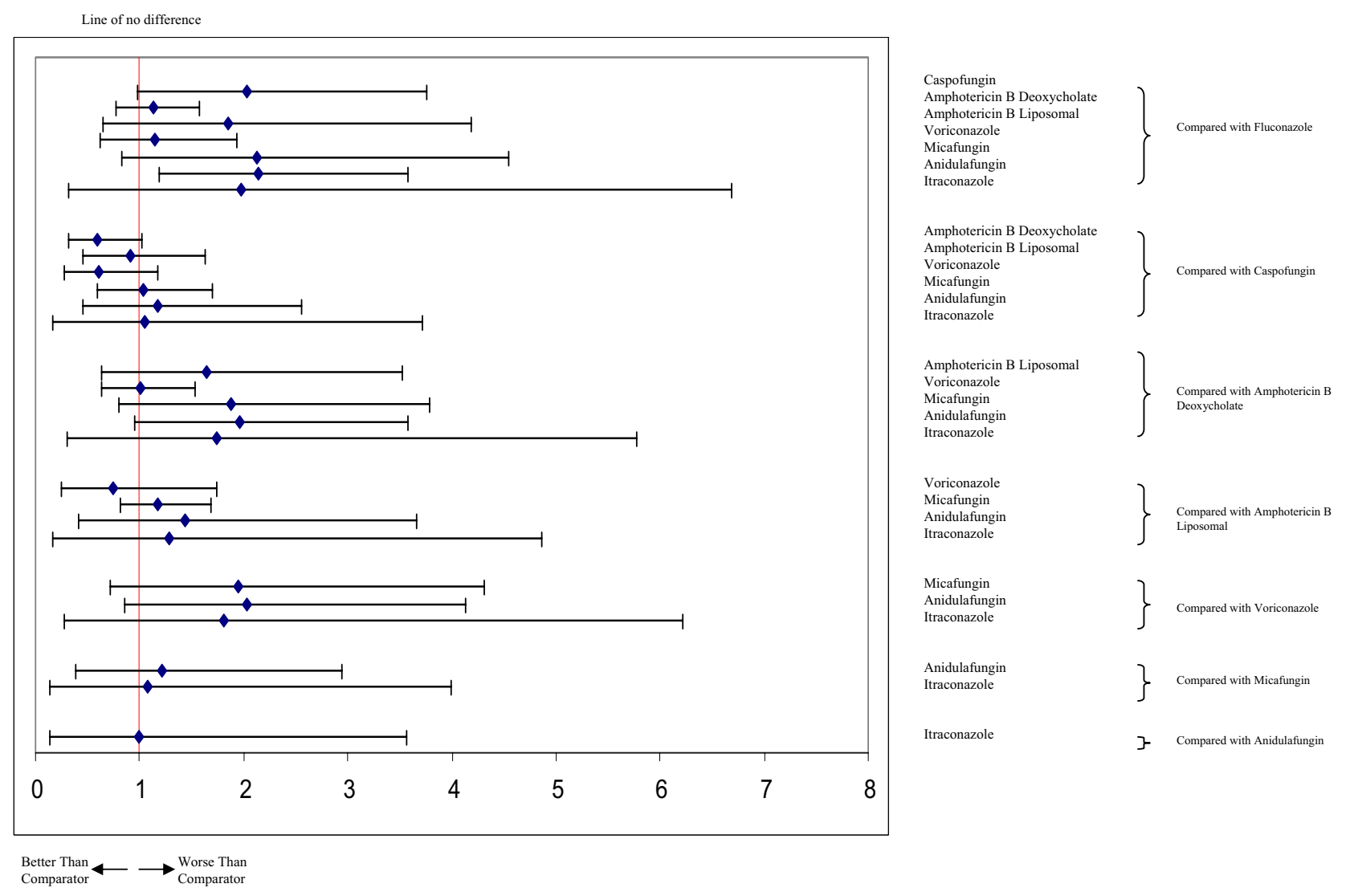

\section{Figure 5}

Caterpillar plots of the odds ratios and $95 \%$ Crls for mixed treatment comparisons, response rates.

a pooled RR of 0.49 (95\% CI, 0.37-0.66, $\mathrm{P}=<0.0001)$ in favour of the echinocandins. Micafungin and caspofungin were not dissimilar in their safety profiles (RR 0.94, 95\% CI, 0.70-1.29). We found no significant difference between anidulafungin versus fluconazole (RR 0.90, 95\% CI, 0.60-1.36, P = 0.66).

When we assessed nephrotoxicity defined variably according to the different studies, we pooled 6 trials of azoles compared to amphotericin B. We found a pooled RR of 0.22 (95\% CI, 0.15-0.32, $\mathrm{P}=<0.0001, \mathrm{I}^{2}=74 \%, \mathrm{P}=$ $0.001)$ in favour of azoles. We also pooled 3 echinocandin compared to amphotericin trials and found a pooled RR 0.31 (95\% CI, 0.17-0.57).

Finally, we assessed hepatic enzyme elevations beyond normal. We pooled 3 trials assessing azoles compared to amphotericin B and found a pooled RR of 1.08 (95\% CI, $\left.0.79-1.47, \mathrm{P}=0.64, \mathrm{I}^{2}=0 \%, \mathrm{P}=0.63\right)$. The 2 echinocandin versus amphotericin $\mathrm{B}$ trials yielded a pooled RR of 1.03 (95\% CI, 0.17-6.26). The single anidulafungin versus fluconazole trial found a RR of 0.21 (95\% CI, 0.05$0.83, \mathrm{P}=0.001$ ) in favour of anidulafungin.
Mixed treatment comparisons

Figure 4 displays the geometric distribution of the mixed treatment comparison. Figures 5 and 6 display the caterpillar plots. Table 1 reports the odds ratios of response rates for all the pairwise comparisons of the antifungal treatment regimens and table 2 presents estimates of the absolute efficacy for each treatment, along with the estimated probability that each treatment is best (response rate).

Table 3 reports the odds ratios of all-cause mortality for all the pairwise comparisons of the antifungal treatment regimens and table 4 presents estimates of the absolute efficacy for each treatment, along with the estimated probability that each treatment is best (mortality).

\section{Discussion}

The results of our systematic review and meta-analysis should be of interest to clinicians, policy-makers and patient groups. Our study found similar effects across within-class interventions. Safety profiles indicate that the class of interventions, azoles and echinocandins, offer protection over amphotericin B in terms of adverse events. 


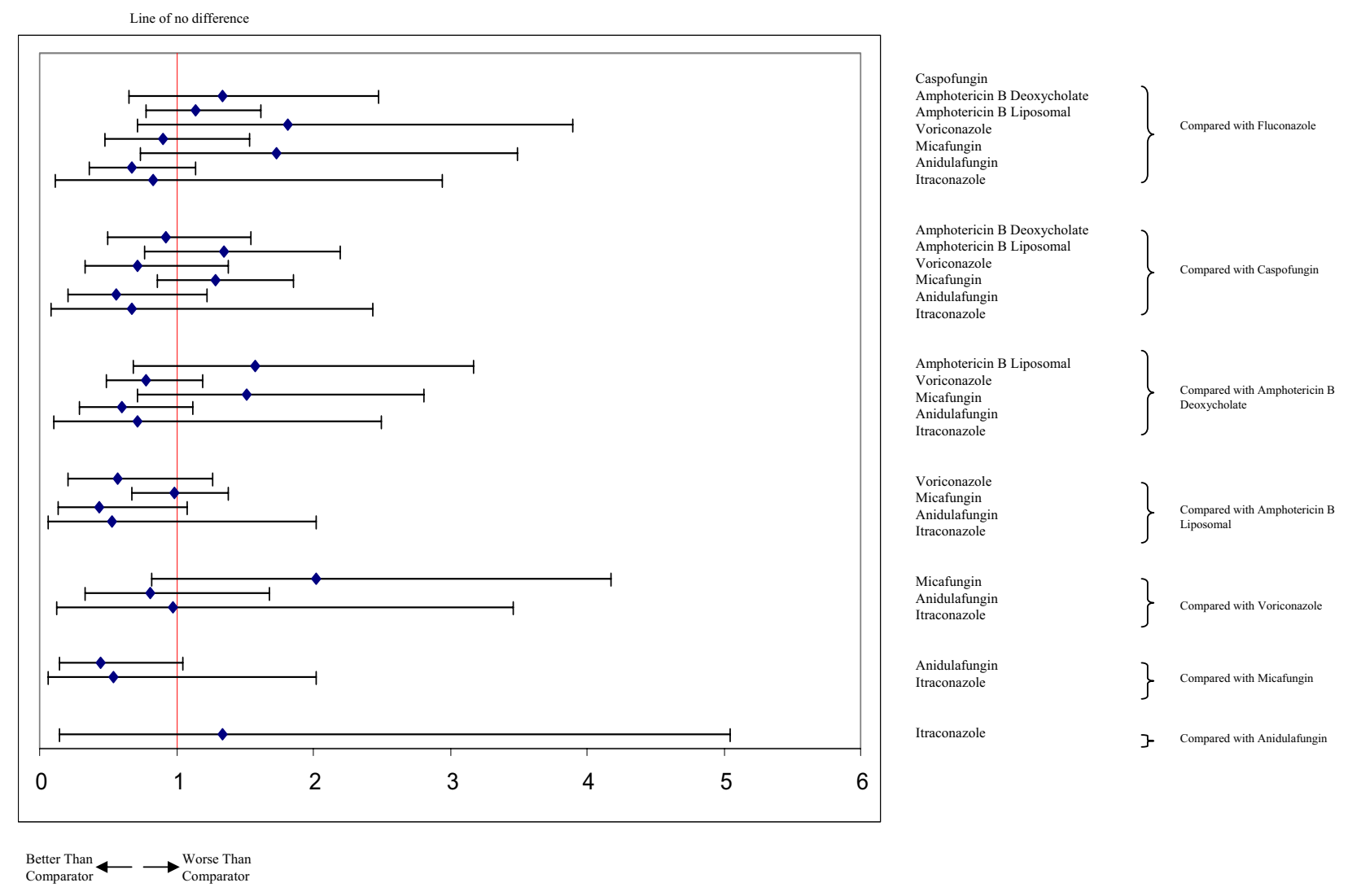

Figure 6

Caterpillar plots of the odds ratios and $\mathbf{9 5 \%}$ Crls for mixed treatment comparisons, all-cause mortality.

There are several important strengths to our meta-analyses that should be considered when interpreting this study. We used extensive searching of electronic databases to identify studies. Thus, we identified more studies than any other systematic reviews $[4,5,25]$. In order to reduce bias, we conducted our searches independently, in duplicate. We extensively searched the bibliographies of published trials and reviews in order to identify unpublished or obscure papers. Finally, we used methodologically advanced approaches to pool and conducted sensitivity analyses across a priori defined covariates.

There are also several limitations to consider when interpreting our analysis. Despite our extensive searching, it is possible that we were unable to identify unpublished trials. Indeed, this issue affects every meta-analysis. We attempted contact through email with 12 study authors to address inclusion and methodological questions but received responses from only 4 ; a common occurrence with systematic reviews [26]. We examined 3 major clinical outcomes and their sub-categories: global response, mortality and adverse events. It is possible that other outcomes would yield differing effects. We conducted mixed treatment comparisons and demonstrated similar within- class effects of drugs. These comparisons provide compelling comparisons, but only head-to-head trials will provide stronger inferences[27,28].

We considered response according to the original papers' definition of response. We considered response according to the original papers' definition of response. Notably, there is substantial variability in the timing and definitions of response across trials which may limit comparability. Timing of assessments ranged from 7 days after start of therapy[2] to up to 12 weeks after the end of therapy[19], with multiple variations in between. The criteria for response also included a wide variety of clinical and microbiological response definition, as well as consideration of the ability to tolerate randomized therapy[2,21,23]. However, we feel our analysis is useful and as we saw no evidence of discrepancies based on our sensitivity analysis. More similarity in endpoint definitions in future trials would be useful to facilitate across-trial comparisons and evaluation of future therapies.

There has been an ongoing debate over the quality of industry-funded trials of antifungal agents, predominantly in the empiric and prophylaxis trials [29-31]. This 
Table 3: Odds ratios and $95 \%$ Crls for mixed treatment comparisons of confirmed infection studies, all-cause mortality

\begin{tabular}{|c|c|c|}
\hline Treatment Comparison & Odds Ratio & 95\% Credible Interval \\
\hline Caspofungin vs. Fluconazole & 1.34 & $(0.65,2.48)$ \\
\hline Amphotericin B Deoxycholate vs. Fluconazole & 1.14 & $(0.78,1.62)$ \\
\hline Amphotericin B Liposomal vs. Fluconazole & 1.81 & $(0.7 I, 3.90)$ \\
\hline Voriconazole vs. Fluconazole & 0.90 & $(0.48,1.53)$ \\
\hline Micafungin vs. Fluconazole & 1.73 & $(0.74,3.49)$ \\
\hline Anidulafungin vs. Fluconazole & 0.67 & $(0.36,1.14)$ \\
\hline Itraconazole vs. Fluconazole & 0.83 & $(0.11,2.94)$ \\
\hline Amphotericin B Deoxycholate vs. Caspofungin & 0.92 & $(0.50,1.54)$ \\
\hline Amphotericin B Liposomal vs. Caspofungin & 1.35 & $(0.77,2.20)$ \\
\hline Voriconazole vs. Caspofungin & 0.72 & $(0.33,1.38)$ \\
\hline Micafungin vs. Caspofungin & 1.28 & $(0.86,1.85)$ \\
\hline Anidulafungin vs. Caspofungin & 0.56 & $(0.21,1.22)$ \\
\hline Itraconazole vs. Caspofungin & 0.67 & $(0.08,2.44)$ \\
\hline Amphotericin B Liposomal vs. Amphotericin B Deoxycholate & 1.58 & $(0.68,3.17)$ \\
\hline Voriconazole vs. Amphotericin B Deoxycholate & 0.78 & $(0.49,1.19)$ \\
\hline Micafungin vs. Amphotericin B Deoxycholate & 1.51 & $(0.72,2.81)$ \\
\hline Anidulafungin vs. Amphotericin B Deoxycholate & 0.60 & $(0.29,1.12)$ \\
\hline Itraconazole vs. Amphotericin B Deoxycholate & 0.72 & $(0.10,2.50)$ \\
\hline Voriconazole vs. Amphotericin B Liposomal & 0.57 & $(0.21,1.26)$ \\
\hline Micafungin vs. Amphotericin B Liposomal & 0.98 & $(0.67,1.38)$ \\
\hline Anidulafungin vs. Amphotericin B Liposomal & 0.44 & $(0.13,1.08)$ \\
\hline Itraconazole vs. Amphotericin B Liposomal & 0.53 & $(0.06,2.02)$ \\
\hline Micafungin vs. Voriconazole & 2.02 & $(0.82,4.18)$ \\
\hline Anidulafungin vs. Voriconazole & 0.81 & $(0.33,1.68)$ \\
\hline Itraconazole vs. Voriconazole & 0.97 & $(0.12,3.46)$ \\
\hline Anidulafungin vs. Micafungin & 0.45 & $(0.15,1.05)$ \\
\hline Itraconazole vs. Micafungin & 0.54 & $(0.06,2.02)$ \\
\hline Itraconazole vs. Anidulafungin & 1.34 & $(0.15,5.05)$ \\
\hline
\end{tabular}

contention has predominantly concerned clarifications post-publication, the use of oral amphotericin B compared to intravenous, and the reporting of all-cause mortality compared to cause-specific mortality. In our analysis we have aimed to overcome this discussion through extensive sensitivity analysis, through focusing on confirmed fungal infections, through evaluating relative effectiveness and through presenting both all-cause and causespecific mortality.

A conundrum in evaluating antifungal therapy is assessing fungle attributable mortality. In most trials, the patients enrolled were at a high risk of mortality due to illness. When we assess all-cause mortality, we recognize that many of the patients would have died from their original disease and not fungal infections. Clinical practice may differ from clinical trial procedures as clinicians may reserve more toxic agents (amphotericin B) for salvage therapy of more severely ill patients who failed short courses of other therapies. However, we could not display a difference between these drug classes when we examined deaths attributable to fungal infections.

We examined the impact of different dosage forms of amphotericin $\mathrm{B}$ to determine if their results change and found it did not. A prior systematic review reported a trend towards all-cause mortality benefit and reduction in nephrotoxicity risk in lipid-based formulations of amphotericin B as compared with conventional amphotericin $B,[6]$ however there was no statistically significant difference in efficacy (clinical response) between the dosage formulations. The reasons for discrepancy between allcause mortality and efficacy were likely a result of clinical and methodologic problems. Dosing and duration of therapy of lipid-based formulations vary widely from study to study, making results difficult to interpret. Trial heterogeneity and small sample size also make it difficult to draw conclusions from comparative studies. An earlier 
Table 4: Absolute treatment efficacy and the probability that each treatment is best for all-cause mortality in the mixed treatment comparisons in confirmed infection studies.

\begin{tabular}{lcc}
\hline & \multicolumn{2}{c}{ Mortality } \\
\hline Fluconazole & 28.44 & 0.006 \\
\hline Caspofungin & 33.83 & 0.008 \\
\hline Amphotericin B Deoxycholate & 30.93 & 0.001 \\
\hline Amphotericin B Liposomal & 39.99 & 0.004 \\
\hline Voriconazole & 25.8 & 0.090 \\
\hline Micafungin & 39.16 & 0.001 \\
\hline Anidulafungin & 20.75 & 0.385 \\
\hline Itraconazole & 21.82 & 0.504 \\
\hline
\end{tabular}

systematic review comparing amphotericin dosage forms found no difference in mortality between lipid and conventional formulations[4]. To our knowledge, no definitive high quality randomized controlled trial comparing amphotericin B dosage formulations has been published since the most recent systematic review.

While we did not include pediatric trials in our analysis, results from a recent systematic review assessing antifungal therapy in children with invasive fungal infections reported congruent conclusions[25]. Although not a meta-analysis, the authors' review (including a supplementation with adult studies) led them to conclude that there was no difference in clinical response between different classes of antifungal agents in the treatment of prolonged febrile neutropenia (empiric therapy) or invasive candidal infection. They did report significant differences in toxicity (particularly nephrotoxicity) between classes that favoured azoles and echinocandins over amphotericin B.

In conclusion, our study suggests that azoles and echinocandins are equally effective interventions for treating invasive candidiasis and confirms the Infectious Disease Society of America (IDSA) guidelines[7], that recommends azoles or echinocandins as the first line treatment for Candida infections. Our analysis found similar withinclass effects. Amphotericin B offers an effective, but more toxic alternative.

\section{Competing interests}

Curtis Cooper, Ping Wu, Dan Perri, and Imad Tleyjeh declare no conflict of interest. Edward Mills has consulted to Pfizer Ltd., Glaxo-Smithkline, Korean HIRA, and International Society for Clinical Trials. Peter Phillips has been a consultant for Pfizer, Merck Frosst Canada,, and Hoffmann-La Roche; and is a member of the speakers' bureau for Pfizer, Merck Frosst Canada, and Schering-Plough Pharmaceuticals.

\section{Authors' contributions}

EM, DP, PW, CC, IT conceived of the study. EM, DP, PW, CC, IT developed the study protocol. EM, DP, PW, CC, IT conducted the searches and data abstraction. EM, DP, PW, CC, IT, JN, PP analyzed and/or interpreted the data. EM, DP, PW, CC, IT, JN, PP wrote the initial drafts of the manuscript and approved submission. EM, DP, PW, CC, IT, JN, PP revised the manuscript. All authors read and approved the final manuscript.

\section{Additional material}

\section{Additional file 1}

Characteristics of included studies. Table addressing study populations, interventions and fungal species measured.

Click here for file

[http://www.biomedcentral.com/content/supplementary/14760711-8-23-S1.doc]

\section{Acknowledgements}

The authors thank Dr. Indra Tumur for important critical reviews. We thank Mr. Chris O'Regan for early comments on the protocol and manuscript.

Funding: This study received unrestricted funding from Pfizer Ltd to evaluate antifungal agents. They had no role in the conduct, interpretation or writing of this manuscript.

\section{References}

I. Empiric antifungal therapy in febrile granulocytopenic patients. EORTC International Antimicrobial Therapy Cooperative Group. Am J Med 1989, 86:668-672.

2. Phillips P, Shafran S, Garber G, Rotstein C, Smaill F, Fong I, Salit I, Miller M, Williams K, Conly JM, Singer J, loannou S: Multicenter randomized trial of fluconazole versus amphotericin $B$ for treatment of candidemia in non-neutropenic patients. Canadian Candidemia Study Group. Eur J Clin Microbiol Infect Dis 1997. 16:337-345.

3. Anaissie El, Darouiche RO, Abi-Said D, Uzun O, Mera J, Gentry LO, Williams T, Kontoyiannis DP, Karl CL, Bodey GP: Management of invasive candidal infections: results of a prospective, randomized, multicenter study of fluconazole versus amphotericin B and review of the literature. Clin Infect Dis 1996, 23:964-972.

4. Johansen HK, Gotzsche PC: Amphotericin B lipid soluble formulations vs amphotericin $B$ in cancer patients with neutropenia. Cochrane Database Syst Rev 2000:CD000969.

5. Gafter-Gvili A, Vidal L, Goldberg E, Leibovici L, Paul M: Treatment of invasive candidal infections: systematic review and metaanalysis. Mayo Clin Proc 2008, 83: 101 I-102I.

6. Barrett JP, Vardulaki KA, Conlon C, Cooke J, Daza-Ramirez P, Evans EG, Hawkey PM, Herbrecht R, Marks DI, Moraleda JM, Park GR, Senn $S$, Viscoli C: A systematic review of the antifungal effectiveness and tolerability of amphotericin B formulations. Clin Ther 2003, 25: 1295-1320. 
7. Pappas PG, Rex JH, Sobel JD, Filler SG, Dismukes WE, Walsh TJ, Edwards JE: Guidelines for treatment of candidiasis. Clin Infect Dis 2004, 38: 16I-89.

8. Meade MO, Guyatt GH, Cook RJ, Groll R, Kachura JR, Wigg M, Cook DJ, Slutsky AS, Stewart TE: Agreement between alternative classifications of acute respiratory distress syndrome. Am J Respir Crit Care Med 200I, 163:490-493.

9. Sheehe PR: Combination of log relative risk in retrospective studies of disease. Am J Public Health Nations Health 1966, 56: $1745-1750$.

10. Pocock SJ: Safety of drug-eluting stents: demystifying network meta-analysis. Lancet 2007, 370:2099-2100.

11. Higgins JP, Thompson SG: Quantifying heterogeneity in a metaanalysis. Stat Med 2002, 21:1539-1558.

12. Thompson SG, Higgins JP: How should meta-regression analyses be undertaken and interpreted? Stat Med 2002, 21:1559-1573.

13. Lu G, Ades A: A combination of direct and indirect evidence in mixed treatment comparisons. Stat Med 2004, 23:3 I 05-3 I 24

14. Gelman A, Rubin DB: Inferences from iterative simulation using multiple sequences. Stat Sci 1992, 7 :

15. Brooks SP, Gelman A: Alternative methods for monitoring convergence of iterative simulations. J Computational and Graphical Statistics 1998, 7:434-455.

16. van't Wout JW, Novakova I, Verhagen CA, Fibbe WE, de Pauw BE, Meer JW van der: The efficacy of itraconazole against systemic fungal infections in neutropenic patients: a randomised comparative study with amphotericin B. J Infect I99|, 22:45-52.

17. Abele-Horn M, Kopp A, Sternberg U, Ohly A, Dauber A, Russwurm $W$, Buchinger W, Nagengast O, Emmerling P: A randomized study comparing fluconazole with amphotericin B/5-flucytosine for the treatment of systemic Candida infections in intensive care patients. Infection 1996, 24:426-432.

18. Kujath P, Lerch K, Kochendorfer P, Boos C: Comparative study of the efficacy of fluconazole versus amphotericin B/flucytosine in surgical patients with systemic mycoses. Infection 1993, 21:376-382.

19. Kullberg BJ, Sobel JD, Ruhnke M, Pappas PG, Viscoli C, Rex JH, Cleary JD, Rubinstein E, Church LW, Brown JM, Schlamm HT, Oborska IT, Hilton F, Hodges MR: Voriconazole versus a regimen of amphotericin B followed by fluconazole for candidaemia in nonneutropenic patients: a randomised non-inferiority trial. Lancet 2005, 366: 1435-I442.

20. Kuse ER, Chetchotisakd P, da Cunha CA, Ruhnke M, Barrios C, Raghunadharao D, Sekhon JS, Freire A, Ramasubramanian V, Demeyer I, Nucci M, Leelarasamee A, Jacobs F, Decruyenaere J, Pittet D, Ullmann AJ, Ostrosky-Zeichner L, Lortholary O, Koblinger S, DiekmannBerndt $\mathrm{H}$, Cornely OA: Micafungin versus liposomal amphotericin B for candidaemia and invasive candidosis: a phase III randomised double-blind trial. Lancet 2007, 369:15/9-1527.

21. Mora-Duarte J, Betts R, Rotstein C, Colombo AL, Thompson-Moya L, Smietana J, Lupinacci R, Sable C, Kartsonis N, Perfect J: Comparison of caspofungin and amphotericin $B$ for invasive candidiasis. N Engl J Med 2002, 347:2020-2029.

22. Reboli AC, Rotstein C, Pappas PG, Chapman SW, Kett DH, Kumar D, Betts R, Wible M, Goldstein BP, Schranz J, Krause DS, Walsh TJ Anidulafungin versus fluconazole for invasive candidiasis. $N$ Engl J Med 2007, 356:2472-2482.

23. Rex JH, Bennett JE, Sugar AM, Pappas PG, Horst CM van der, Edwards JE, Washburn RG, Scheld WM, Karchmer AW, Dine AP, et al: A randomized trial comparing fluconazole with amphotericin B for the treatment of candidemia in patients without neutropenia. Candidemia Study Group and the National Institute. N Engl J Med 1994, 33 I: 1325-1330.

24. Pappas PG, Rotstein CM, Betts RF, Nucci M, Talwar D, De Waele I] Vazquez JA, Dupont BF, Horn DL, Ostrosky-Zeichner L, Reboli AC, Suh B, Digumarti R, Wu C, Kovanda LL, Arnold LJ, Buell DN: Micafungin versus caspofungin for treatment of candidemia and other forms of invasive candidiasis. Clin Infect Dis 2007, 45:883-893.

25. Blyth CC, Palasanthiran P, O'Brien TA: Antifungal therapy in children with invasive fungal infections: a systematic review. Pediatrics 2007, I I 9:772-784.

26. Mullan RJ, Flynn DN, Carlberg B, Tleyjeh IM, Kamath CC, LaBella ML, Erwin PJ, Guyatt GH, Montori VM: Systematic reviewers commonly contact study authors but do so with limited rigor. Journal of clinical epidemiology 2009, 62: 138-142.
27. Glenny AM, Altman DG, Song F, Sakarovitch C, Deeks J], D'Amico R, Bradburn M, Eastwood AJ: Indirect comparisons of competing interventions. Health Technol Assess 2005, 9:1-134.

28. Song F, Altman DG, Glenny AM, Deeks JJ: Validity of indirect comparison for estimating efficacy of competing interventions: empirical evidence from published meta-analyses. Bmj 2003 , 326:472.

29. Johansen HK, Gøtzsche PC: Problems in the design and reporting of trials of antifungal agents encountered during metaanalysis. JAMA 1999, 282: I752-1759.

30. Glasmacher A, Prentice A, Gorschlüter M, Engelhart S, Hahn C, DjuIbegovic B, Schmidt-Wolf IGH: In Reply. I Clin Oncol 2005, 23:9429-9432.

31. Gotzsche PC, Johansen HK: Misleading statements in industrysponsored meta-analysis of itraconazole. J Clin Oncol 2005, 23:9428-9429.
Publish with Bio Med Central and every scientist can read your work free of charge

"BioMed Central will be the most significant development for disseminating the results of biomedical research in our lifetime. "

Sir Paul Nurse, Cancer Research UK

Your research papers will be:

- available free of charge to the entire biomedical community

- peer reviewed and published immediately upon acceptance

- cited in PubMed and archived on PubMed Central

- yours - you keep the copyright
BioMedcentral 